\title{
Hydrogen sulfide and nonalcoholic fatty liver disease
}

\author{
Panzhi Wang ${ }^{1}$, Liming $\mathrm{Wu}^{2,3,4}$ \\ ${ }^{1}$ Center of Medical Journals, ${ }^{2}$ Division of Hepatobiliary and Pancreatic Surgery, Department of Surgery, ${ }^{3}$ Key Laboratory of Precision Diagnosis and \\ Treatment for Hepatobiliary and Pancreatic Tumor of Zhejiang Province, the First Affiliated Hospital, College of Medicine, Zhejiang University, \\ Hangzhou 310003, China; ${ }^{4}$ Collaborative Innovation Center for Diagnosis and Treatment of Infectious Diseases, Hangzhou 310003 , China \\ Correspondence to: Liming Wu, MD, PhD. Division of Hepatobiliary and Pancreatic Surgery, Department of Surgery, First Affiliated Hospital, \\ College of Medicine, Zhejiang University, 79 Qingchun Road, Hangzhou 310003, China. Email: wlm@zju.edu.cn.
}

Submitted Feb 26, 2018. Accepted for publication Mar 05, 2018.

doi: 10.21037/hbsn.2018.03.03

View this article at: http://dx.doi.org/10.21037/hbsn.2018.03.03

Nonalcoholic fatty liver disease (NAFLD) is one of the most common chronic liver disease worldwide. The septum of NAFLD ranges from simple steatosis to nonalcoholic steatohepatitis, the latter of which may further progress to liver cirrhosis and even more advanced stages in $20 \%$ patients $(1,2)$. During last decade, nonalcoholic steatohepatitis has been identified as the most rapidly growing indication for liver transplantation in patients with hepatocellular carcinoma in the United States (3). Meanwhile, NAFLD also significantly increases risk of cardiovascular diseases, type 2 diabetes, chronic kidney disease, and colorectal neoplasia.

The pathogenesis of NAFLD has not been fully clarified (4). Hydrogen sulfide has been recognized as a toxic gas with the smell of rotten eggs for centuries. Recent studies (5-8) found that hydrogen sulfide regulates variety of physiological processes such as vasorelaxation, neuromodulation, and inflammatory responses. The liver is a major organ for endogenous hydrogen sulfide production and clearance, and there are increasing evidences indicating that hydrogen sulfide plays significant roles in physiology and pathophysiology of liver diseases (5). An early study found that endogenous hydrogen sulfide synthesis was impaired in cirrhotic livers (6). Subsequent studies found that hydrogen sulfide protected against hepatic ischemia/ reperfusion injury and carbon tetrachloride-induced liver injury in rats $(7,8)$.

The effects of hydrogen sulfide on regulation of hepatic lipid metabolism and NAFLD have also been intensively investigated. A recent study reported that hepatic hydrogen sulfide was significantly lower in the high fat diet-fed mice than that of controls (9). Another recent study reported similar results that hepatic hydrogen sulfide biosynthesis was impaired in methionine and choline deficient diet-induced rat model of NAFLD (10). Further mouse experiments showed that treatment with sodium hydrosulfide, a hydrogen sulfide donor, prevents nonalcoholic steatohepatitis by abating oxidative stress and suppressing inflammation (11). These studies highlight significant roles of hydrogen sulfide in NAFLD.

The reason for why hydrogen sulfide synthesis is impaired in NAFLD remains unclear. Hydrogen sulfide can be produced by pyridoxal-5'-phosphate (PLP)dependent or PLP-independent enzymes in mammalian tissues. Cystathionine $\beta$-synthase (CBS) and cystathionine $\gamma$-lyase (CSE) are PLP-dependent enzymes, and 3-mercaptopyruvate sulfurtransferase (MPST) is a PLPindependent enzyme utilizing 3-mercaptopyruvate as substrate to generate hydrogen sulfide (12). All these three enzymes express in the liver and regulate liver functions via hydrogen sulfide production. CBS/CSE system is considered to be responsible for the majority of endogenous hydrogen sulfide production, while MPST plays the aided action during hydrogen sulfide synthesis (13). It has been reported that the CBS/CSE system is highly expressed and active in the liver and has been proposed to serve as a potential therapeutic target for NAFLD (14). Nevertheless, the role of MPST in NAFLD has not been fully understood.

In a recent issue of Gut, Li et al. reported that hepatic MPST was significantly up-regulated in NAFLD patients 
with predominant expression in steatotic hepatocytes (15). High fat diet-feeding or free fatty acids treatment also significantly up-regulated MPST expression in hepatocytes. Although MPST was up-regulated in the livers of high fat diet-fed mice and in free fatty acidsstimulated hepatocytes, hepatic hydrogen sulfide synthesis was impaired in these NAFLD models. In addition, knockdown of hepatic MPST significantly increased rather than inhibited hydrogen sulfide synthesis, and markedly ameliorated hepatocyte steatosis both in vivo and in vitro, while overexpression of MPST induced the opposite effects. Another important finding of Li's study is that their co-immunoprecipitation study found that MPST directly interacted with and negatively regulated CSE. Their mechanism experiments provided further evidences that hydrogen sulfide mediated the processing of suppressing SREBP-1 pathway, weakening JNK1 signaling and ameliorating hepatic oxidative stress, thereby contributing to the improvement of hepatocyte steatosis. These results suggested a significant role of MPST in NAFLD, and implied that MPST may be a potential therapeutic target for NAFLD.

It is undoubtful that hydrogen sulfide plays critical role in the pathogenesis of NAFLD. The study by Li et al. provided evidences for the first time that MPST, a PLP-independent enzyme catalyzing hydrogen sulfide production, is significantly involved in regulation of NAFLD. NAFLD includes a wide range of conditions spanning from simple steatosis to nonalcoholic steatohepatitis and even more severe stages. The current studies mainly focused on the role of hydrogen sulfide in simple steatosis. It would be of extreme importance to conduct further study to better elucidate the role of hydrogen sulfide and its catalyzing enzymes in the development and progression of NAFLD.

\section{Acknowledgements}

Funding: This study was supported by the Key Research Development Program of Zhejiang Province (No. 2018C03018), the Traditional Chinese Medicine Scientific Research Fund Project of Zhejiang Province (No. 2017ZA079).

\section{Footnote}

Conflicts of Interest: The authors have no conflicts of interest to declare.

\section{References}

1. Younossi ZM, Koenig AB, Abdelatif D, et al. Global epidemiology of nonalcoholic fatty liver disease-Metaanalytic assessment of prevalence, incidence, and outcomes. Hepatology 2016;64:73-84.

2. Kim HY, Kim CW, Park CH, et al. Low skeletal muscle mass is associated with non-alcoholic fatty liver disease in Korean adults: the Fifth Korea National Health and Nutrition Examination Survey. Hepatobiliary Pancreat Dis Int 2016;15:39-47.

3. Younossi ZM. Nonalcoholic fatty liver disease and nonalcoholic steatohepatitis: Implications for liver transplantation. Liver Transpl 2018;24:166-70.

4. Shen F, Zheng RD, Sun XQ, et al. Gut microbiota dysbiosis in patients with non-alcoholic fatty liver disease. Hepatobiliary Pancreat Dis Int 2017;16:375-81.

5. Mani S, Cao W, Wu L, et al. Hydrogen sulfide and the liver. Nitric Oxide 2014;41:62-71.

6. Fiorucci S, Antonelli E, Mencarelli A, et al. The third gas: $\mathrm{H} 2 \mathrm{~S}$ regulates perfusion pressure in both the isolated and perfused normal rat liver and in cirrhosis. Hepatology 2005;42:539-48.

7. Kang K, Zhao M, Jiang H, et al. Role of hydrogen sulfide in hepatic ischemia-reperfusion-induced injury in rats. Liver Transpl 2009;15:1306-14.

8. Tan G, Pan S, Li J, et al. Hydrogen Sulfide Attenuates Carbon Tetrachloride-Induced Hepatotoxicity, Liver Cirrhosis and Portal Hypertension in Rats. PLoS ONE 2011;6:e25943.

9. Peh MT, Anwar AB, Ng DS, et al. Effect of feeding a high fat diet on hydrogen sulfide (H2S) metabolism in the mouse. Nitric Oxide 2014;41:138-45.

10. Luo ZL, Tang LJ, Wang T, et al. Effects of treatment with hydrogen sulfide on methionine-choline deficient diet-induced non-alcoholic steatohepatitis in rats. J Gastroenterol Hepatol 2014;29:215-22.

11. Wu D, Zheng N, Qi K, et al. Exogenous hydrogen sulfide mitigates the fatty liver in obese mice through improving lipid metabolism and antioxidant potential. Med Gas Res 2015;5:1.

12. Shibuya N, Tanaka M, Yoshida M, et al. 3-Mercaptopyruvate sulfurtransferase produces hydrogen sulfide and bound sulfane sulfur in the brain. Antioxid Redox Signal 2009;11:703-14.

13. Kabil O, Vitvitsky V, Xie P, et al. The quantitative significance of the transsulfuration enzymes for H2S production in murine tissues. Antioxid Redox Signal 
2011;15:363-72.

14. Sarna LK, Siow YL, O K. The CBS/CSE system: a potential therapeutic target in NAFLD? Can J Physiol Pharmacol 2015;93:1-11.

Cite this article as: Wang $\mathrm{P}, \mathrm{Wu} \mathrm{L}$. Hydrogen sulfide and nonalcoholic fatty liver disease. HepatoBiliary Surg Nutr 2018;7(2):122-124. doi: 10.21037/hbsn.2018.03.03
15. Li M, Xu C, Shi J, et al. Fatty acids promote fatty liver disease via the dysregulation of 3-mercaptopyruvate sulfurtransferase/hydrogen sulfide pathway. Gut 2017. [Epub ahead of print]. 